Baudrimont theilt die Analyse seines Lycoperdon gigantenm (S. Journ. de pharm. et de chim. Janvr. 1867) mit, welcher $0,78 \% \mathrm{~N}$ als Ammoniak und $46,58 \% \mathrm{C}$. enthält. $\mathrm{Zn}$ seiner Entwickelung in 14 Tagen hatte dieser Schwamm täglich 18,9 Liter $\mathrm{CO}^{2}$ absorbirt, im Ganzen 37,8 Liter Luft und bildete nach Berechnung 12,061 Zellen in der Secunde! (Journ. de pharm. et de chim.).

R.

\title{
Notiz über die Cultur der Cinchonen In Brittisch Indien und ther Chinarindenproben ron dort auf der Pariser Ausstellung 186\%.
}

Von J. L. Soubeiran und Augustin Delondre.

Im April 1859 hatte sich Lord Stanley entschieden, Herrn Cl. R. Markham nach Peru zu schicken, um Cinchonasamen und Pflanzen zu sammeln; letzterer verliess England im December desselben Jahres; am 2े8. Juli 1860 war er wieder dahin zurückgekehrt; am 28. Ootober langte er in Ootacamund an. Trotz der Schwierigkeiten eines solchen Unternehmens und ungeachtet der kurzen Zeit, welche seitdem verflossen ist, beträgt die Zahl der Cinchonas, welche sich im Gouvernement Madras im April 1866 in voller Erde befanden, d. h. also vor mehr als 2 Jahren, 1 Million 123,625 Stück. (Nach einer Mittheilung Markham's mögen sie sich gegen Ende 1866 auf 1,500,000 Stück belaufen haben). Alle diese Pflanzen waren in einem durchaus günstigen Vegetationszustande, blühten und setzten wohlentwickelte Früchte an. Andere Anpflanzungen wurde in dem W ynaad errichtet, in dem Coorg, auf den Pulneybergen und in Travancore, namentlich zu Peermede, in der Präsidentschaft Madras; im brittischen Sikkim und zu Darjeeling, in der Präsidentschaft Bengalen; in dem Thale von Kangra, im Punjab; zu Lingmulla, in der Praesidentsch. Bombay, und zu Paradenia, auf der Insel Ceylon. In allen diesen Oertlichkeiten scheint ein voller Erfolg die Anstrengungen der Pflanzer gekrönt zu haben. Auf Ceylon besonders scheint sich die werthvollste Art auf die genügendste Weise zu entwickeln. - Dieses glückliche Resultat scheint in erster Linie der Wahl der Gegend zu verdanken, welche streng dem natürlichen Standort der Cinchonen in Süd-Amerika entspricht und in 2. Linie der gründlichen Kenntniss and dem Verdienste 
des H. Mac Ivor, Oberaufseher der. Cinchonapfianzungen des englischen Gouvernements in Indien.

In jeder dieser angegebenen Oertlichkeiten hat das englische Gouvernement der Privatindustrie und selbst den Rajah's, den Eingeborenen des Landes Stecklinge abgeben können und diese Versuche scheinen gute Hoffnung auf Erfolg zu versprechen.

Kurz, schon im April 1866 gab es schon über 2 Millionen im Freien cultivirte Cinchonaptlanzen auf den verschiedenen Punkten von brittisch Indien und aller Wahrscheinlichkeit nach wird diese Zahl sich während der letzten 2 Jahre noch beträchtlich vermehrt haben.

Die Herren J. E. How ard u. Sohn haben zur Pariser Ausstellnng 186710 Proben verschiedener Rinden gesendet, welche von den zu Ootacamund cultivirten Cinchonen stammen, ferner eine Chinarindenprobe auf Ceylon cultivirt, endlich ein höchst interessantes Rindenstiick von $\mathrm{Cinchona}$ nitida, von einem in den Gewächshäusern des Herrn J. E. Howard in England cultivirten Exemplare dieser Cinchona. Dieses Exemplar ist nicht allein in botanischer, sondern auch in chemischer Hinsicht merkwürdig, da es beweist, dass die Cinchonen, überall wo sie auch wachsen, in ihrer Rinde Chinin enthalten, ein wichtiger Punkt in Betreff der Acclimatisation derselben.

Herr Mac Ivor hat sehr wichtige Beobachtungen in Betreff der Wirkung der Cultur auf die Quantität und selbst auf die Naturder in der Rinde enthaltenen Alkaloide gemacht; er constatirte, dass durch eine passende Cultur der Gehalt der Rinde an Alkaloiden auf eine wahrhaft beträchtliche Weise, in den Verhältnissen von $1: 2$, 1:3 und selbst in einer bedeutenderen Proportion vermehrt werden könne, namentlich durch eine Bedeckung der Baumstämme mit Moos, und dass mit dieser Vorsicht die abgenommene Rinde sich aufs neue erzeugt, ähnlich wie der Kork an den Korkeichen des Südens.

Zur Seite der indischen Cinchonarinden hatten die Herren Howard auf der Pariser Ausstellung 1867 schöne Proben von Chinin und schwefelsaurem Chinin, aus indobrittischen Chinarinden gewonnen, aufgestellt. Eine der grössten Sehenswürdigkeiten ihrer Ausstellung war die Sammlung von mehr als 150 Species Chinarinden des Handels, wahrer und falscher, aus ihrem Privatmuseum zu Stratford, wo sich die ausgezeichnete Fabrik chemischer Produkte befindet. Die Rinden waren begleitet von 70 Proben von Salzen des Chinins, Chinidins, 
Cinchonins und Cinchonidins, welche die Verschiedenheiten und die Aehnlichkeiten zeigten, die zwischen diesen 4 Alkaloïden statt finden. Mit Ausnahme der schwefels. Salze zeigte die Mehrzahl dieser Salze eine grosse Neigung $\mathrm{C}$ i nchonaroth 'unter dem Einflusse des Lichtes zu bilden. Diese Beobachtung stimmt vollkommen mit den Resultaten der fortgesetzten Versuche des Herrn Mac Ivor zu Ootacamund, im Betreff der B em o os ung (moussage) der Rinden.

Das brittis che Gouvernement hatte zur Ausstellung 1867 noch andere Cinchonarindenproben gesendet, welche von den Pflanzungen zu Ootacamund stammten und als Sendung des H. W. G. M a c I v or sich in der speciellen Ausstellung der britt. Indien befanden.

Wir bemerken schliesslich, dass die Herren Markham und Mac Ivor und alle diejenigen, welche ihnen beigestanden, sich nicht allein um ihr Land, sondern auch um die Menschheit wohl verdient gemacht haben, indem sie dazu beitrugen, ein so nützliches Arzneimittel fortzupflanzen; dabei müssen wir hervorheben, dass auch die Herren $H o w a r d$ und Hooker durch ibre wissenschaftlichen Kenntnisse zu diesem Zwecke wesentlich mit beigetragen haben. (Journ. $d$. pharm. et d. chim. Déc. 1867. V. Sér. t. VI. p. $432-435$.). H. $L$.

\section{Indisches Gummi.}

Lobeuf und Dumenil machen anf eine als indisches Gummi vorkommende Waare aufmerksam, die zuweilen als arabisches Gummi verkauft wird, aber in der Pharmacie als solches nicht angewendet werden kann; in der Industrie dient es zur Appretur der Zeuche.

Dieses Gummi kommt in Kisten von ungefähr 4 Centnern über London aus Indien; man findet es gemengt mit Thränen von verschiedener Nuance und mit Unreinigkeiten. In Frankreich sucht man die weissesten Thränen davon aus und mengt sie unter das arabische oder. Senegal-Gummi oder man verkauft es auch ganz für dieses.

Bei genauer Prüfung der neuen Waare bemerkt man, dass sie weniger durchsichtig ist als das arabische Gummi; ihre Oberfläche ist weniger zersprungen, sie ist glänzend und oft warzenförmig; auch ist das neue Gummi weniger zerreiblich als das arabische. Aber sein Hauptunterscheidungs- 